\title{
First case of extensively drug-resistant tuberculosis treated with both delamanid and bedaquiline
}

\author{
To the Editor:
}

The European Respiratory Journal has recently discussed delamanid and bedaquiline and their use in difficult-to-treat cases affected by multidrug-resistant tuberculosis (MDR-TB) or extensively drug-resistant (XDR-TB) [1-4]. The use of delamanid or bedaquiline is particularly important when four active drugs cannot be identified and included in a regimen, as per World Health Organization (WHO) guidelines [1-6]. Recently a debate has been initiated around the report of a severe, almost untreatable, XDR-TB case who could not access both new drugs simultaneously [7-10] due to concerns about possible additive toxicity (cardiotoxicity), as well as the lack of evidence and specific guidance on their combined use [10-13].

In the meantime some essential criteria have been proposed [10] which, if met, might allow for the co-administration of delamanid and bedaquiline to be appropriate and safe: an effective treatment cannot be designed by using only one new drug in addition to the optimised background regimen as per WHO guidelines; the clinical centre is qualified and equipped to monitor the patient during treatment; informed consent and pharmacovigilance are in place; and use of the new drugs is supported by expert opinion (e.g. the TB Consilium or similar bodies; www.tbconsilium.org) $[5,10]$. This approach aims to increase the chance of treatment success, while minimising the patients' risk of adverse events and ensuring their timely management if they appear. Furthermore, it allows reduction of the risk of transmission of a potentially lethal strain [10] and prevents resistance to the new drugs.

This study reports the rationale for prescribing both delamanid and bedaquiline in an XDR-TB case and describes the difficulties encountered in the early phase of treatment.

The patient, a 39 year-old female Tibetan refugee living in India, who was HIV negative, had a TB relapse in 2011 after being declared cured twice, in 1997 and 2005, respectively. Based on drug susceptibility testing (DST), she was prescribed second-line drugs. Following 12 months of second-line drug treatment, her sputum culture was persistently positive; she was subsequently referred to Delek Hospital, Dharamshala, India. A new DST showed resistance to isoniazid, rifampicin, kanamycin, ethionamide, para-aminosalicylic acid (PAS), ofloxacin, moxifloxacin, amikacin and capreomycin (table 1). Despite different drug combinations, which included all five WHO classes of anti-TB drugs, the patient required a total of 38 months of anti-TB drugs to finally obtain culture conversion in the last year of treatment, which reverted 2 months after the suspension of the drugs.

At this stage the treating physician requested the inclusion of the patient in the delamanid compassionate use programme via the TB Consilium platform; based on DST, the patient had XDR-TB with a single certified active drug, clofazimine. DSTs for ethambutol, pyrazinamide, streptomycin, cycloserine/terizidone and meropenem/clavulanic acid were not available, while all other drugs tested showed resistance. The patient had bilateral upper zone fibrocavitary lesions on chest radiography and poor lung reserve ruling out the possible benefit of surgical resection.

In such a setting, the combination of clofazimine and delamanid without a third (and fourth) active drug may potentially have resulted in delamanid resistance. Given there were no other available drugs to design an effective regimen for this patient, the TB Consilium experts recommended the simultaneous introduction of delamanid and bedaquiline in combination with an optimised background regimen. Therefore, both new drugs were requested and made available to make an effective salvage regimen.

The regimen included delamanid and bedaquiline (at the recommended doses of $200 \mathrm{mg}$ and $400 \mathrm{mg}$, respectively), clofazimine $(200 \mathrm{mg})$ and terizidone $(1 \mathrm{~g})$ and meropenem ( $1 \mathrm{~g}$ three times daily) plus amoxicillin/clavulanate ( $1 \mathrm{~g} / 200 \mathrm{mg}$ three times daily, intravenous), all started on February 25, 2016 [6]. The patient's baseline ECG, serum albumin and potassium were normal, and there was no history of cardiac disease or comorbidities predisposing cardiac dysfunction. Moxifloxacin was not considered in the regimen as DST showed resistance to high-dose moxifloxacin, and adding this to regimen already containing three QT (ECG Q-T wave interval) prolonging drugs would have increased the risk of adverse events.

On March 4, 2016 the treating physician, concerned about QT corrected with heart rate (QTc) prolongation in his patient, consulted the TB Consilium again. The patient was asymptomatic, with a baseline QTc (prior to delamanid and bedaquiline administration) of $<450 \mathrm{~ms}$, thereafter increasing to 
TABLE 1 Clinical characteristics of the first case treated with both delamanid and bedaquiline

Details

Comments

Country of birth
Age
Sex
Body weight at diagnosis ${ }^{\#}$
Case category
Number of previous anti-TB
treatments
Drugs administered in previous
anti-TB treatments

India

39 years

Female

$65 \mathrm{~kg}$

Retreatment case

4

Kanamycin 750 mg i.m. (12 months)

Levofloxacin $1 \mathrm{~g}$, PAS $10 \mathrm{~g}$, cycloserine $750 \mathrm{mg}$, ethionamide

$750 \mathrm{mg}$, capreomycin $1 \mathrm{~g}$ i.m. (14 months)

High-dose isoniazid $900 \mathrm{mg}$, rifabutin $300 \mathrm{mg}$, clofazimine

$200 \mathrm{mg}$, clarithromycin $1 \mathrm{~g}$, amoxicillin/clavulanate $625 \mathrm{mg}$,

terizidone $1 \mathrm{~g}$ three times daily, imipenem $500 \mathrm{mg}$ i.v. three

times daily (12 months), linezolid $600 \mathrm{mg}$ then $300 \mathrm{mg}$

Previous outcome

Cured (twice)

$26.9 \mathrm{~kg} \cdot \mathrm{m}^{-2}$

Preveline

Bacteriology at baseline

Sputum smear positive

Culture positive

Xpert positive

At Day 18: smear negative

Culture taken after 28 days

of treatment: ongoing

(negative on the 14th day

of MGIT culture)

Radiology

Drug resistances

Last treatment regimen
Bilateral upper zones fibrocavitary lesions

Resistant to 12 drugs: Isoniazid, rifampicin, kanamycin, amikacin, capreomycin, moxifloxacin, ofloxacin, ethionamide, PAS, linezolid, high-dose isoniazid, high-dose moxifloxacin Susceptible to: clofazimine

Delamanid, bedaquiline, clofazimine $(200 \mathrm{mg})$, terizidone $(1 \mathrm{~g})$ and meropenem $1 \mathrm{~g}$ three times daily plus amoxicillin/ clavulanate $1 \mathrm{~g} / 200 \mathrm{mg}$ three times daily i.v., all started on February 25, 2016
Bedaquiline stopped on March 7, 2016, restarted March 12, 2016

TB: tuberculosis; PAS: para-aminosalicylic acid; i.m.: intramuscular; i.v.: intravenous; MGIT: mycobacteria growth indicator tube. ${ }^{\#}$ : August $31,2015$.

$476 \mathrm{~ms}$ after five doses and to $486 \mathrm{~ms}$ on March 3, 2016 (after eight doses of the new drugs). Serum potassium was $3.5 \mathrm{mmol} \cdot \mathrm{L}^{-1}$ on March 5, 2016 (day 10 of treatment) supplemented with oral potassium chloride, and been within the normal range on weekly follow-up.

Given the ongoing concerns and debate on QTc prolongation with the combination of delamanid, bedaquiline and clofazimine $[11,12]$, both the TB Consilium experts advised to stop bedaquiline (which was carried out on March 7, 2016) and to continue close patient monitoring.

The following management plan was recommended by the TB Consilium experts:

1) Repeat ECG biweekly and closely monitor the QTc interval.

2) Monitor urea and electrolytes weekly and rule out hypokalaemia, hypomagnesaemia, hypocalcaemia, etc. (which could potentially prolong QTc). Correct the electrolyte imbalance if necessary.

3) Repeat the thyroid function test to rule out hypothyroidism, which could potentially prolong QTc because of long-term exposure to ethionamide and PAS, and manage hypothyroidism accordingly.

4) If QTc increases to $>500 \mathrm{msec}$, stop the whole regimen and then continue with biweekly ECG monitoring. It was unfortunately impossible to consider stopping clofazimine in absence of alternatives. Preliminary evidence in South Africa shows that the mean increase in QTc within a 2 month period using bedaquiline was $\sim 8 \mathrm{msec}$ (N. Ndjeka; National Dept of Health, Pretoria, South Africa; personal communication).

5) Once QTc is $<500 \mathrm{msec}$, re-introduce the regimen and monitor ECG weekly/biweekly or monthly depending on the QTc levels.

In the same round of consultations, suggestions from the TB Consilium experts included:

1) Restart bedaquiline and continue the current regimen while observing QTc, and if above $500 \mathrm{msec}$, stop clofazimine. 
2) Restart bedaquiline and continue the current regimen, monitor ECG and consider adding verapamil to control QTc. Verapamil is an agent that may negate the effect of bedaquiline and clofazimine on QTc; however, there is little evidence supporting this [14].

3) Should the patient become symptomatic (for example syncope or arrhythmias), consider a definitive stop of bedaquiline, unless implantation of a defibrillator device is possible.

Following the TB Consilium experts' recommendations, electrolytes were corrected, verapamil (40 mg three times daily) was added on March 12, 2016 and bedaquiline restarted, as QTc was still $<500 \mathrm{~ms}$ (489 ms). After five additional days (day 21 of treatment), QTc was $479 \mathrm{~ms}$ and the patient remained asymptomatic. Bedaquiline was then reduced to $200 \mathrm{mg}$ three times weekly. ECG repeated at day 27 reported a QTc of $491 \mathrm{msec}$. The patient remains asymptomatic. Electrolytes and serum albumin are in the normal range. As far as treatment response is concerned, the sputum smear was negative after 18 days of treatment, while the sputum culture taken after 28 days was still negative on the 14 th day of mycobacteria growth indicator tube culture.

This experience emphasises the importance of conducting clinical trials on the combined use of delamanid and bedaquiline (e.g. those planned by the National Institute of Allergy and Infectious Diseases; https:// clinicaltrials.gov/ct2/show/NCT02583048), whose results will, unfortunately, not be available for the next 2-3 years. In the meantime, in cases of extremely severe clinical conditions that may be life-threatening and when recourse to other second-line drugs is extremely limited, it is our opinion that associating the two drugs would be possible, provided that this is done in a properly qualified MDR-TB treatment centre with adequate facilities to closely monitor the patient's condition and drug safety, and that the patient is duly informed of the limited evidence available (i.e. signed informed consent) [10]. The support of the TB Consilium or similar platforms can help ensure appropriate use of the new drugs and close monitoring during their co-administration [10].

Furthermore, the experience of this first case treated with both drugs shows that if the safety criteria mentioned above are met as in this case [7], co-administration of delamanid and bedaquiline would be possible, although the clinical management of these cases can be demanding, expensive and complicated [15].

@ERSpublications

Report of the first case, concerns and challenges of treatment of severe XDR-TB with both delamanid and bedaquiline http://ow.ly/WzeB3004Cmo

Marina Tadolini ${ }^{1,7}$, Rangjung Dolma Lingtsang ${ }^{2,7}$, Simon Tiberi ${ }^{3,7}$, Martin Enwerem ${ }^{4,7}$, Lia D'Ambrosio ${ }^{5,6,7}$, Tsetan Dorji Sadutshang ${ }^{2}$, Rosella Centis ${ }^{5}$ and Giovanni Battista Migliori ${ }^{5}$

${ }^{1}$ Unit of Infectious Diseases, Dept of Medical and Surgical Sciences, Alma Mater Studiorum University of Bologna, Bologna, Italy. ${ }^{2}$ Delek Hospital, Dharamshala, India. ${ }^{3}$ Division of Infection, Barts Health NHS Trust, London, UK. ${ }^{4}$ Amity Health Consortium, Johannesburg, South Africa. ${ }^{5}$ Fondazione S. Maugeri, Care and Research Institute, Tradate, Italy.

${ }^{6}$ Public Health Consulting Group, Lugano, Switzerland. ${ }^{7}$ These authors contributed equally.

Correspondence: Giovanni Battista Migliori, Fondazione S. Maugeri, Care and Research Institute, Via Roncaccio 16, 21049, Tradate, Italy. E-mail: giovannibattista.migliori@fsm.it

Received: March 302016 | Accepted after revision: April 192016 | First published online: June 102016

This article is one of a small annual allocation selected for open access publication at the discretion of the editor.

The authors alone are responsible for the views expressed in this publication and they do not necessarily represent the decisions and policies of their institutions.

Conflict of interest: None declared.

\section{References}

1 Tiberi S, D’Ambrosio L, De Lorenzo S, et al. Tuberculosis elimination, patients' lives and rational use of new drugs: revisited. Eur Respir J 2016; 47: 664-667.

2 Pontali E, Sotgiu G, D'Ambrosio L, et al. Bedaquiline and multidrug-resistant tuberculosis: a systematic and critical analysis of the evidence. Eur Respir J 2016; 47: 394-402.

3 Sotgiu G, Pontali E, Centis R, et al. Delamanid (OPC-67683) for treatment of multi-drug-resistant tuberculosis. Expert Rev Anti Infect Ther 2015; 13: 305-315.

4 Wells CD, Gupta R, Hittel N, et al. Long-term mortality assessment of multidrug-resistant tuberculosis patients treated with delamanid. Eur Respir J 2015; 45: 1498-1501.

5 Esposito S, D'Ambrosio L, Tadolini M, et al. ERS/WHO Tuberculosis Consilium assistance with extensively drug-resistant tuberculosis management in a child: case study of compassionate delamanid use. Eur Respir J 2014; 44: 811-815.

6 Tiberi S, Payen MC, Sotgiu G, et al. Effectiveness and safety of meropenem/clavulanate-containing regimens in the treatment of multidrug and extensively drug-resistant tuberculosis. Eur Respir J 2016; 47: 1235-1243.

7 Reed C, Mason L, Cox H, et al. Compassionate and optimum use of new tuberculosis drugs. Lancet Infect Dis 2015; 15: 1131 
8 Migliori GB, Sotgiu G, Gandhi NR, et al. Drug resistance beyond extensively drug-resistant tuberculosis: individual patient data meta-analysis. Eur Respir J 2013; 42: 169-179.

9 Migliori GB, De Iaco G, Besozzi G, et al. First tuberculosis cases in Italy resistant to all tested drugs. Euro Surveill 2007; 12: E070517.1.

10 Matteelli A, D’Ambrosio L, Centis R, et al. Compassionate and optimum use of new tuberculosis drugs. Lancet Infect Dis 2015; 15: 1131-1132.

11 World Health Organization. The use of delamanid in the treatment of multidrug-resistant tuberculosis. Interim policy guidance. WHO/HTM/TB2014.23. Geneva, World Health Organization, 2014.

12 World Health Organization. The use of bedaquiline in the treatment of multidrug-resistant tuberculosis. Interim policy guidance. WHO/HTM/TB/2013.6. Geneva, World Health Organization, 2013.

13 Migliori GB, Lienhardt $\mathrm{C}$, Weyer $\mathrm{K}$, et al. Ensuring rational introduction and responsible use of new TB tools: outcome of an ERS multisector consultation. Eur Respir J 2014; 44: 1412-1417.

14 Srikrishna G, Gupta S, Dooley KE, et al. Can the addition of verapamil to bedaquiline-containing regimens improve tuberculosis treatment outcomes? A novel approach to optimizing TB treatment. Future Microbiol 2015; 10: $1257-1260$.

15 Guglielmetti L, Le Dû D, Jachym M, et al. Compassionate use of bedaquiline for the treatment of multidrug-resistant and extensively drug-resistant tuberculosis: interim analysis of a French cohort. Clin Infect Dis 2015; 60: 188-194.

\section{Compassionate use of new drugs in children and adolescents with multidrug-resistant and extensively drug-resistant tuberculosis: early experiences and challenges}

To the Editor:

The World Health Organization (WHO) estimated that 480000 new multidrug-resistant (MDR) tuberculosis (TB) cases occurred globally in 2014, with 190000 deaths. Limited data are available on the burden of MDR-TB in children. A recent systematic review estimated that 32000 children acquire MDR-TB annually; of these, very few are correctly diagnosed and provided with appropriate treatment [1].

Treatment of drug-resistant TB is long, expensive and associated with frequent adverse events [1-5]. In children, treatment is further complicated by limited data on appropriate dosing and safety, and a lack of child-friendly formulations. New anti-TB drugs are urgently needed to improve treatment tolerability and outcome, particularly for MDR-TB cases with additional second-line drug resistance, for whom identifying at least four active drugs is difficult with the current armamentarium of drugs [1, 4-11]. Two novel anti-TB drugs, delamanid $[6,8,12]$ and bedaquiline $[6,7,9,10,12]$, have received conditional approval for use in adults with MDR-TB. While the WHO interim guidance does not include a recommendation on their use in children due to lack of data, the Centers for Disease Control and Prevention guidelines state that bedaquiline use can be considered for children when treatment options are limited.

A number of paediatric clinical trials are ongoing or planned. The Otsuka 232 (phase 1, pharmacokinetics and safety to determine the appropriate dose for MDR-TB in children) and 233 (phase 2, 6-month safety, efficacy and pharmacokinetics trial in children with MDR-TB) trials of delamanid have begun recruitment. Preliminary pharmacokinetics and safety data in 6-17-year-old children from these trials have been presented, showing an excellent safety profile and leading to weight-based dosing recommendations for patients of $20-35 \mathrm{~kg}$ to receive half the adult dose (50 mg of delamanid twice daily) (presented in poster format (A-960) by J. Hafkin; Pharmacokinetics and safety of delamanid in paediatric MDR-TB patients, ages 6-17 years; San Diego, Interscience Conference on Antimicrobial Agents and Chemotherapy (ICAAC), 2015). A follow-up study to confirm the long-term safety, tolerability and pharmacokinetics of delamanid in these age groups and younger children (0-5 years) is ongoing and data are not yet available (presented in poster format (EP-115-04) by J. Hafkin; Long-term safety, tolerability and pharmacokinetics of delamanid in paediatric MDR-TB patients, ages 12-17 years; Cape Town, The 46th Union World Conference on Lung 